\title{
Self-Ordered Front under Temporally Irregular Forcing: Ratchet-Like Transport of the Quasi-Periodically Forced Front
}

\author{
R. BAKAnAS ${ }^{a, *}$, F. IVANAUSKAS ${ }^{b, c}$ AND V. JASAitis ${ }^{b}$ \\ ${ }^{a}$ Semiconductor Physics Institute, Center for Physical Sciences and Technology \\ A. Goštauto 11, LT-01108 Vilnius, Lithuania \\ ${ }^{b}$ Faculty of Mathematics and Informatics, Vilnius University, Naugarduko 24, LT-03225 Vilnius, Lithuania \\ ${ }^{c}$ Institute of Mathematics and Informatics, Akademijos 4, 2600 Vilnius, Lithuania
}

(Received July 22, 2010)

\begin{abstract}
Ratchet-like transport of the quasi-periodically forced "bistable" front joining two states of the different stability in the reaction-diffusion system is considered by use of the piecewise linear rate (reaction) function of the reaction kinetics. We approximate the oscillatory force acting on the front in the system by the bi-harmonic forcing functions being a superposition of the single-harmonic components (the Fourier modes) of the different frequencies, either commensurate or incommensurable ones. By considering the response of the self-ordered front to the oscillatory forces used we analyze the effect of the temporally irregular oscillations of the ac forcing on the ratchet-like shuttling of the ac driven front. By comparing the average characteristics of the spurious drift derivable in both cases of the periodically and quasi-periodically forced fronts we show that the temporally irregular fluctuations of the oscillatory force shrink the spurious drift of the front. More specifically, we find the performance of the ratchet-like shuttling of the self-ordered fronts is much lesser pronounced with the quasi-periodic, temporally irregular ac forcing if compared to that derivable by the rigorously periodic forcing, in both cases of the symmetrical and asymmetrical rate functions satisfying the different symmetry. The average characteristics of the spurious drift, that describe the dependence of the mean drift velocity of the ac driven front versus both the amplitude (strength) and the frequency of the oscillatory forces used are presented.
\end{abstract}

PACS: 05.45.-a, 02.30.Jr, 82.40.Ck

\section{Introduction}

Spatio-temporal control of both the point-particles (electrons, atoms, molecules) and self-ordered fronts without any net external force, under the generic name of "ratchet-like transport", has today become a fundamental field of multidisciplinary research in a wide variety of the systems ranging from biology to physics (e.g., see Refs. [1] and [2-9], respectively, and references therein). The ratchet-like transport implies that the oscillatory force of zero-time average, either noisy or regular one, is converted into the directed net motion of the particle/ self-ordered front. The ordinary ratchets performing the unforced shuttling of the point particles operate in the systems lacking the spatial inversion (reflection) symmetry. An intriguing feature of the systems performing the ratchet-like transport of the self-ordered fronts, both coherent and dissipative ones, is their capability to operate even in the case of the spatially uniform system satisfying a continuous symmetry with respect to the infinitesimal translations of the spatial coordinate. Importantly, the presence of dissipation, which leads to the temporal irreversibility of the dynamical equations, is a crucial factor for the existence of the unforced dc motion of the self-ordered fronts. The dissipation effaces the memory of

* corresponding author; e-mail: bakanas@pfi.lt the system when the system evolves in time, i.e., the memory of the initial conditions of the ac driven front gets lost as the development of the system progresses.

In the present report we deal with the dissipative fronts, namely, the unforced dc motion of the "bistable" fronts (BFs) joining two states of the different stability, the stable and metastable one, in a bistable system of the reaction-diffusion type is considered. The ratchet-like transport of the dissipative fronts that result from the competition between the nonlinearity and diffusion in the essentially dissipative systems of the reaction-diffusion type has been studied in numerous papers, in both cases of the noisy (randomly fluctuating) and deterministic (periodically oscillating) zero-mean ac forces (e.g., see Refs. [3-8] and [9-12], respectively). Two different mechanisms underlying the spurious drift discussed have been identified, namely, the parametrically stimulated and directly induced dc drift of the fronts (see Refs. $[3-5]$ and $[6-12]$, respectively). The parametrically stimulated dc drift, more exactly, the ratchet-like transport derivable by the multiplicative forcing function in the governing equation of the ac driven front comes through the action of the external oscillatory field of zero-time average on the externally controllable parameter of the system: the external time-symmetric field acting on the system is converted (transformed) into the asymmetrically oscillating force of a finite time average, acting on the front in the system, as a result, the "unforced" uni- 
directional net motion of the ac driven front takes place. By contrast, the directly induced dc drift, namely, the ratchet-like transport derivable by the additive forcing $f(t)$ implies that the average force acting on the front in the system equals zero. The governing equation of the directly driven $\mathrm{BF}$ reads

$$
u_{t}-u_{z z}-c(t) u_{z}+R(u)=f(t),
$$

where the function $u(z, t)$ denotes the step-like field of the front propagating at the instantaneous velocity $c(t)$, and the notation $z=x-\chi(t)$ stands for the traveling (comoving) coordinate, where $\chi(t)=\int_{t_{0}}^{t} \mathrm{~d} t^{\prime} c\left(t^{\prime}\right)$, with $t_{0}$ denoting the time moment, at which both the traveling coordinate and that being at rest, $z$ and $x$, respectively, coincide. The rate function $R(u)$, which characterizes the rate of the transient processes in the system, has three zeroes at $u=u_{1}, u_{2}, u_{3}$ (say $u_{1}<u_{2}<u_{3}$ ), more specifically, the following relations hold: $R^{\prime}\left(u_{1,3}\right)>0$ and $R^{\prime}\left(u_{2}\right)<0$ where the prime denotes the derivative. The outer zero-points of the rate function, $u_{1}$ and $u_{3}$, describe two steady spatially uniform states of the system, the stable and metastable one, and the inner $u_{2}$ stands for unstable one. Without loss of generality, we shall take in what follows that $u_{0}(z \rightarrow-\infty) \rightarrow u_{1}$, and $u_{0}(z \rightarrow \infty) \rightarrow u_{3}$.

The ratchet-like transport of $\mathrm{BF}$ implies that the oscillatory speed function $c(t)$ of the ac driven front is split into two parts: (a) an oscillatory part of zero time average, and (b) a systematic part denoting the mean drift velocity of the ac driven front, i.e., the "speed rectification" of the ac driven BF takes place. Broadly speaking, the "speed rectification" is a nonlinear transformation process, which converts the oscillatory "input" function $f(t)$ of zero-time average into the "output" (response) function $c(t)$ of a finite time average. The mechanism of the speed rectification discussed, more exactly, the general principle of how the oscillatory force of zero-time average is converted into the speed function of a finite time average was discussed in Ref. [12]. Symmetry analysis that was performed with the rate function of the general form in the governing equation of the slowly (quasi-stationary) driven BF demonstrated that the ac driven front experienced the speed rectification discussed if the rate function was asymmetrically shaped with respect to the inner zero-point, namely, when the inequality $R\left(u-u_{2}\right) \neq-R\left(u_{2}-u\right)$ was satisfied. Evidently, the performance of the ratchet-like shuttling discussed should be sensitive to the peculiarities of $f-t$ dependence (e.g., see Refs. [11-14]). In particular, it depends on the temporal symmetry of the ac force acting on the front in the system; the average $v-f_{\mathrm{a}}$ characteristics, which describe the dependence of the mean drift velocity $v$ of the ac driven BF versus the amplitude $f_{\mathrm{a}}$ of the oscillatory force $f(t)$, derivable in two different cases of the time-symmetric and time-asymmetric forcing functions satisfying the different temporal symmetry radically differ, as shown in Refs. [9-11] (see also Refs. [14], for related studies).
In the present report the ratchet-like transport of the quasi-periodically forced BFs that are influenced by the temporally irregular ac force being characterized by the incommensurate "frequency mixing" of the single-harmonic components (the Fourier modes) is considered. Namely, we approximate the oscillatory force $f(t)$ acting on the front in the system by the bi-harmonic forcing function being a linear combination (sum) of the single-harmonic components with the incommensurate, rationally independent frequencies. The multi-harmonic oscillatory forces $f(t)$ that are characterized by the incommensurate "frequency mixing" of the single-harmonic components exhibit the temporally irregular behavior. Namely, the "amplitude" (strength) of the quasi-periodic forcing $f(t)$, more specifically, the deviation of the oscillatory function $f(t)$ between two points being separated by the time interval $\Delta t$ irregularly oscillates with $\Delta t$. We notice that the quasi-periodic forcing functions of zero-time average have been used in the studies of the ordinary ratchets, as simplified approximations (emulations) of the noisy, randomly fluctuating forces (see Refs. [15]).

A random force may be presented by a superposition of the single-harmonic modes being characterized by the randomly distributed frequencies, both the commensurate and incommensurate ones. In particular, the Gaussian random function of zero mean and delta correlated is characterized by the compact and infinitely broad frequency spectrum (e.g., see Refs. [16]). It should be noted that the unforced dc drift of the randomly "wandering" BFs that are influenced by the noisy, temporally irregular ac forces representing both cases of the "white" (uncorrelated) and colored (correlated) noises has been studied in Refs. [5-7]. More specifically, the direct calculations being performed within both the cubic polynomial and sine-Gordon models of the system, by use of the perturbation techniques demonstrated that a white Gaussian noise was not capable to induce the directed net motion of $\mathrm{BF}$; the spurious drift of the randomly wandering $\mathrm{BF}$ disappeared in both cases of the symmetrical (symmetrically shaped) and asymmetrical (asymmetrically shaped) the rate functions satisfying the different $R-u$ symmetry (see below), if the random force $f(t)$ was Gaussian and delta correlated. Both the colored noise and the broken $R-u$ symmetry of the rate function with respect to the inner zero-point $u_{2}$ are both crucial factors for the existence of the directed net motion of the randomly wandering BF, as shown in Ref. [6]. It should be noted that the noise-supported propagation of the self-ordered fronts propagating into metastable state of the bistable system was observed experimentally in the system of the coupled electronic elements (nonlinear resonators) (see Ref. [17]).

Obviously, the results derivable within the perturbation theory are of limited utility; they fit well in the case of the weak forcing only. The noise-assisted propagation of BFs in the random fields of the higher intensities, more specifically, the ratchet-like transport of BFs, derivable at the higher levels of the noise has not been studied so far. Thus, it is not clear whether the previous results 
being derived within the perturbation theory will hold at the higher levels of the noise, beyond the perturbative approaches discussed. Clearly, the quasi-periodic forcing, which may be treated as the deterministic "equivalent" (emulation) of the random force, is very drastic simplification of the noisy driver. Nevertheless, it provides us with a possibility to learn whether or not, and how strongly the temporally irregular oscillations of the ac force acting on the front in the system are capable support the propagation of BFs. In addition, the employment of the quasi-periodic forcing is meaningful for its simplicity. The "efficiency", the quasi-periodic driver, in terms the spurious drift discussed, is the principal goal of the present study. By comparing the average characteristics of the spurious drift derivable in both cases of the periodically and quasi-periodically forced BFs we investigate whether the temporally irregular ac driver results in a lower, or inversely, in a higher performance of the ratchet-like shuttling of BFs, if compared to that derivable by the rigorously periodic driver. As already noted, the spurious drift of BFs is sensitive to the symmetry of the rate function. In view of this, we shall deal the piecewise linear rate function of the flexible $R-u$ symmetry, encompassing both cases of the symmetrical and asymmetrical rate functions. The ratchet-like transport of the quasi-periodically forced BFs, as far as we know, has not been studied so far.

The paper is organized as follows. In Sect. 2 we discuss the model and the techniques used. Section 3 deals with the ratchet-like shuttling of the quasi-periodically forced BFs. The spurious drift of BFs is sensitive to the "rate" (frequency) of the oscillatory force acting on the front in the system. In view of this, in considering the ratchet-like transport of BFs we distinguish between two regimes of the slow and fast oscillatory forces. The particular case of the slow driving forces that induce almost immediate, nearly instantaneous response of $\mathrm{BF}$ to the applied forcing, is studied in Sect. 3.1, and the other case of the fast driving, namely, the rectified oscillatory motion of the self-ordered fronts with a delayed response of BF to the applied forcing is studied in Sect. 3.2. Finally, in Sect. 4 we summarize the main results.

\section{Model and techniques used}

\subsection{Model}

We approximate the rate function by the piecewise linear dependence

$$
R(u)=\left\{\begin{array}{l}
\alpha_{1}\left(u-u_{1}\right), \quad u<u_{\mathrm{M}}, \\
-\alpha_{2}\left(u-u_{2}\right), \quad u_{\mathrm{M}}<u<u_{\mathrm{m}}, \\
\alpha_{3}\left(u-u_{3}\right), \quad u>u_{\mathrm{m}},
\end{array}\right.
$$

where the free, adjustable parameters $u_{i}$ and $\alpha_{i}$ satisfy the relations $u_{1}<u_{\mathrm{M}}<u_{2}<u_{\mathrm{m}}<u_{3}$ and $\alpha_{i}>0(i=1,2,3)$. The extremes of the rate function, $R_{\mathrm{M}} \equiv R\left(u_{\mathrm{M}}\right)$ and $R_{\mathrm{m}} \equiv R\left(u_{\mathrm{m}}\right)$, are given by the relation $R_{\mathrm{M}, \mathrm{m}}=\alpha_{2}\left(u_{2}-u_{\mathrm{M}, \mathrm{m}}\right)$ where the subscripts $\mathrm{M}$ and $\mathrm{m}$ denote "Maximal" and "minimal", respectively. In what follows we shall deal with the self-similar rate functions being defined by the relation

$$
R(u ; C):=R_{0}(u)+C,
$$

where $C$ is the free constant and the subscript zero refers to the balanced rate function $R_{0}(u)$ satisfying Maxwellian construction. The Maxwellian construction is balanced (satisfied) if the equality $S_{0} \equiv$ $\int_{u_{1}}^{u_{3}} \mathrm{~d} u R_{0}(u)=0$ holds where the notation $S_{0}$ stands for the total area subtracted by $R_{0}-u$ dependence within the interval $\left[u_{1}, u_{3}\right]$ of the variable $u$. For our purposes it is useful to introduce the "balance parameter" $h_{\mathrm{R}}$ of the rate function being defined by the relation $h_{\mathrm{R}}:=-R_{\mathrm{M}} / R_{\mathrm{m}} \equiv-\left(R_{0 \mathrm{M}}+C\right) /\left(R_{0 \mathrm{~m}}+C\right)$ where by $R_{0 \mathrm{M}}$ and $R_{0 \mathrm{~m}}$ we denote the extremes of the balanced rate function. By tuning the balance parameter $h_{\mathrm{R}}$ one governs Maxwellian construction of the rate function $R(u ; C)$, as a consequence, one arrives at the different propagation rates $c_{0}$ of the free, unperturbed $(f(t) \equiv 0)$ $\mathrm{BF}$. The free $\mathrm{BF}$ is static $\left(c_{0}=0\right)$ if the rate function is balanced, namely, if one takes that $h_{\mathrm{R}}=h_{0}$, where

$$
h_{0}=\sqrt{\left(1+r_{3}^{-1}\right) /\left(1+r_{1}^{-1}\right)},
$$

and the quantity, $r_{1,3}=\alpha_{1,3} / \alpha_{2}$, denotes the slope parameter of the rate function. The free $\mathrm{BF}$ is running $\left(c_{0} \neq 0\right)$ if the rate function is unbalanced, namely, if one takes that $h_{\mathrm{R}} \neq h_{0}$.

As previously mentioned, the symmetry of the rate function plays a prominent role, in considering the ratchet-like shuttling of BFs (see Refs. [10, 12]). Two different families of the symmetrical and asymmetrical rate functions $R(u ; C)$ may be typified. The rate function $R(u ; C)$ is symmetrical (symmetrically shaped) if the basic (balanced) rate function $R_{0}(u)$ is rigorously symmetric, namely, if the equality holds,

$$
R_{0}\left(u-u_{2}\right)=-R_{0}\left(u+u_{2}\right) \text {. }
$$

By contrast, the asymmetrical rate function $R(u ; C)$ implies that

$$
R_{0}\left(u-u_{2}\right) \neq-R_{0}\left(u+u_{2}\right) .
$$

The piecewise linear rate function (2.1) is of the flexible $R-u$ symmetry: by taking $\alpha_{1}=\alpha_{3}$ and $\alpha_{1} \neq \alpha_{3}$ one arrives at both cases of the symmetrical and asymmetrical rate functions $R(u ; C)$, respectively. Both the heights of the self-similar rate functions, $\Delta R=R_{\mathrm{M}}\left(u_{\mathrm{M}} ; C\right)-$ $R_{\mathrm{m}}\left(u_{\mathrm{m}} ; C\right)$, and the positions of the extremes being located at $u=u_{\mathrm{M}}$ and $u=u_{\mathrm{m}}$ do not depend on the free constant $C$. From Eq. (2.1), in the conjunction with (2.2), it follows that:

$$
\begin{aligned}
& R_{\mathrm{M}}=h_{\mathrm{R}}\left(1+h_{\mathrm{R}}\right)^{-1} \Delta R, \\
& R_{\mathrm{m}}=-\left(1+h_{\mathrm{R}}\right)^{-1} \Delta R, \\
& u_{1}=u_{\mathrm{M}}-\alpha_{1}^{-1} R_{\mathrm{M}}, \quad u_{2}=u_{\mathrm{M}}-\alpha_{2}^{-1} R_{\mathrm{M}}, \\
& u_{3}=u_{\mathrm{m}}-\alpha_{3}^{-1} R_{\mathrm{m}} .
\end{aligned}
$$

As discussed, by tuning the balance parameter $h_{\mathrm{R}}$ of the rate function $R(u ; C)$ one arrives at the different prop- 
agation rates of the free $\mathrm{BF}$. The maximal propagation rate of the forward $\left(c_{0}>0\right)$ and the backward $\left(c_{0}<0\right)$ running BF is achieved by taking the limits $h_{\mathrm{R}} \rightarrow \infty$ and $h_{\mathrm{R}} \rightarrow 0$, respectively. More specifically, one has that (a) $c_{0} \rightarrow c_{\mathrm{M}}$ if $h_{\mathrm{R}} \rightarrow \infty$, and (b) $c_{0} \rightarrow-c_{\mathrm{M}}$ if $h_{\mathrm{R}} \rightarrow 0$ where the notation $c_{\mathrm{M}}$ stands for the marginal velocity of $\mathrm{BF}$, namely, one has that $c_{\mathrm{M}}=2 \sqrt{\alpha_{2}}$. In closing the discussion of the rate functions used we notice that the free front-solutions of BFs, $u_{0}(z)$, derivable by the piecewise linear rate function (2.1) are presented in Ref. [18]. Let us turn to the forcing functions.

\subsection{Forcing functions}

In what follows we shall deal with the biharmonic forcing functions described by the following expression:

$$
f(t)=f_{0}\left(1+b_{\mathrm{F}}\right)^{-1}\left[\sin \omega t+b_{\mathrm{F}} \sin (\mu \omega t+\Delta \varphi)\right]
$$

where by $b_{\mathrm{F}}, \mu, \Delta \varphi$, and $f_{0}$ we denote the free, adjustable parameters. The presented expression encompasses both cases of the periodic and quasi-periodic forcing functions below labeled by $f_{P}(t)$ and $f_{Q}(t)$, respectively. By taking the free parameter $\mu$ to be the rational/irrational number one arrives at the periodic/quasi-periodic forcing function. The "efficiency" of the temporally irregular ac driver is the central subject of the present study. Obviously, in order to evaluate the effect of the temporally irregular oscillations of the quasi-periodic forcing on the performance of the ratchet-like shuttling of BFs, the characteristic parameters of both oscillatory functions $f_{Q}(t)$ and $f_{P}(t)$ must be properly chosen. Namely, the frequencies and the amplitude-to-frequency ratios of the single-harmonic components of both forcing functions $f_{Q}(t)$ and $f_{P}(t)$ should be close to each other. We shall demand in what follows that $b_{\mathrm{F}}=1$ and $\Delta \varphi=\pi$, and the parameter value $\mu$ being related to the forcing functions discussed, $f_{Q}(t)$ and $f_{P}(t)$, is taken as follows: $\mu=\sqrt{3}$ and $\mu=2$, respectively. Thus, both the "spectral content" and the amplitude-to-frequency ratios of the single-harmonic components of both forcing functions used are close to each other. The amplitude (maximal deviation)

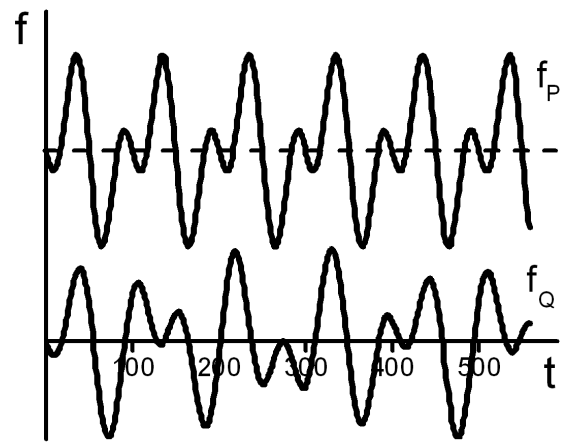

Fig. 1. The periodic $\left(f_{P}\right)$ and quasi-periodic $\left(f_{Q}\right)$ forcing functions. The period of the basic mode of both oscillatory functions was taken as follows: $T=100$. $f_{\mathrm{a}}$ of the oscillatory force $f(t)$ is another important parameter of the ac driver. Referring to the forcing functions $f_{Q}(t)$ and $f_{P}(t)$ we get, respectively, that

$$
f_{\mathrm{a}}=f_{0},
$$

and

$$
f_{\mathrm{a}}=\frac{f_{0}}{64}(30+2 \sqrt{33})^{1 / 2}(3+\sqrt{33}) \approx 0.88 f_{0} .
$$

Clearly, the amplitude $f_{\mathrm{a}}$ of the oscillatory force acting on the front cannot exceed the critical value, $f_{\mathrm{M} x} \equiv$ $\min \left\{R_{\mathrm{M}},-R_{\mathrm{m}}\right\}$, above which the global stability of the ac driven BF breaks down. Thus, we shall demand in what follows that $f_{\mathrm{a}}<f_{\mathrm{M} x}$. Both forcing functions $f_{Q}(t)$ and $f_{P}(t)$ are depicted in Fig. 1, for comparison. Let us touch briefly on the techniques used.

\subsection{Approximations and techniques used}

In what follows we shall deal with the scaled speed functions $s(t)$ and the scaled forcing functions $f^{*}(t)$ being defined by the relations, $s(t):=c(t) / c_{\mathrm{M}}$ and $f^{*}(t):=$ $f(t) / \Delta R$, respectively. The scaled functions are preferable over the ordinary ones; the average characteristics of the spurious drift of BFs are not sensitive to the height $\Delta R$ of the self-similar rate functions $R(u ; C)$ if the scaled functions discussed are used, as shown in Refs. [10, 12]. To simplify the denotations, we shall drop the asterisk in the denotation $f^{*}(t)$, namely, in what follows by $f(t)$ we shall denote the scaled forcing function. Let us turn to the techniques used. The governing Eq. (1.1) is analytically not solvable. In view of this, we used both the numerical simulations and analytic tools. Namely, the particular case of the quasi-stationary (quasi-statically slowly) driven BFs that instantly follow the ac force, without any time delay, was studied by use of the quasi-stationary equation

$$
\begin{aligned}
& u_{z z}+c_{\mathrm{M}} s_{\mathrm{A}}(t) u_{z}-R_{\mathrm{F}}(u)=0, \\
& R_{\mathrm{F}}(u)=R(u)-f(t),
\end{aligned}
$$

where the notation $s_{\mathrm{A}}(t)$ stands for the "instantaneous" speed function. The presented equation (the adiabatic approximation of the governing Eq. (1.1)) fits well for the low driving "rates", when the period $T$ of the ac force acting on the front significantly exceeds the characteristic relaxation time of the system, $\tau_{\mathrm{R}}=\max \left\{\alpha_{1}^{-1}, \alpha_{3}^{-1}\right\}$ (see Ref. [19]). The instantaneous speed function $s_{\mathrm{A}}(t)$ is derivable analytically, by use of the "speed equation" being derived by the direct solution of the quasi-stationary Eq. (2.8) (see Refs. [10, 18]). The speed equation reads

$$
\frac{\Psi_{S n}\left[s_{\mathrm{A}}(t)\right]}{\exp \left(-\varphi\left[s_{\mathrm{A}}(t)\right]\right) \sin \Phi\left(s_{\mathrm{A}}(t)\right)}=\frac{h_{\mathrm{R}}-\left(1+h_{\mathrm{R}}\right) f(t)}{1+\left(1+h_{\mathrm{R}}\right) f(t)},
$$

where the auxiliary quantities $\Psi_{S n}\left(s_{\mathrm{A}}\right), \varphi\left(s_{\mathrm{A}}\right)$ and $\Phi\left(s_{\mathrm{A}}\right)$ are functions of both the slope coefficients $\left\{\alpha_{i}\right\}$ and the speed function $s_{\mathrm{A}}(t)$ which we seek. Regretfully, the explicit expressions of the auxiliary functions $\Psi_{S n}\left(s_{\mathrm{A}}\right)$, $\varphi\left(s_{\mathrm{A}}\right)$ and $\Phi\left(s_{\mathrm{A}}\right)$ are rather involved; the auxiliary func- 
tions are transcendental. They are presented in Appendix. In considering the ratchet-like shuttling of BFs under the slow, quasi-stationary zero-mean ac forces $f_{Q}(t)$ and $f_{P}(t)$ we used the speed Eq. (2.9).

The other case of the fast driving, more specifically, the front dynamics with the delayed response of $\mathrm{BF}$ to the applied forcing was studied numerically, by the direct solution of the governing Eq. (1.1). To find both the front-solution $u(z, t)$ and the speed function $s(t)$ of the ac driven $\mathrm{BF}$ we numerically simulated the drift motion of the ac driven front. Equation (1.1) was approximated by the finite difference scheme in the co-moving coordinates, $z=x-x_{C}(t)$, with the traveling center of the ac driven front, $x_{C}(t)$, being defined by the relation $x_{C}(t)=\Delta u^{-1}(t) \int_{-\infty}^{\infty} \mathrm{d} x x u_{x}(x, t)$, where $\Delta u(t)=\int_{-\infty}^{\infty} \mathrm{d} x u_{x}(x, t)$. The actual (retarded) speed functions $s(t)$ that follow behind the ac force with some retardation have been obtained by considering the traveling center $x_{C}(t)$ of the front.

\section{Self-ordered front under temporally irregular ac forcing: ratchet-like transport of the quasi-periodically forced fronts}

\subsection{Low-frequency response: self-ordered front under slow oscillatory force}

We begin our discussion of the ratchet-like shuttling of the quasi-periodically forced BFs with the particular case of the slow oscillatory forces satisfying the relation $\mu \omega \tau_{\mathrm{R}} \ll 1$. The propagation velocity $s(t)$ of the ac driven BF almost instantly follows the ac force if the frequency of the fastest (super-harmonic) mode of the multi-harmonic forcing function is much lesser if compared to the characteristic relaxation rate of the system, $\tau_{\mathrm{R}}^{-1}$ (e.g., see Refs. [19, 20]). Furthermore, the slow oscillatory force usually maximizes the spurious drift of BF. In particular, the periodic and extremely slow (quasi-stationary) ac force of zero-time average is of the highest "efficiency" in terms of the spurious drift discussed, namely, it always induces the maximal shift of the mean drift velocity of the ac driven BF, as shown in Ref. [20] (for related experimental studies, see Ref. [13]). Nevertheless, the temporal behavior of both forcing functions used, the periodic and quasi-periodic one, radically differs. As previously noted, the spurious drift of BFs is very sensitive to the peculiarities of $f-t$ dependence; thus, it is not clear a priori, in advance whether and how strongly the temporally irregular ac forcing $f_{Q}(t)$ is capable to support the propagation of BF.

As discussed, the "efficiency" of the quasi-periodic, temporally irregular ac driver, in terms of the spurious drift discussed, is the central subject of the present study. The "efficiency" of the oscillatory force of zero-time average may be evaluated by considering the acceleration factor, $\rho:=\left|s_{0}\right|^{-1} v$, which denotes the relative increase of the mean drift velocity of the ac driven of BF. The average $\rho-f_{\mathrm{a}}$ characteristics of the periodically and quasi-periodically forced BFs that are derived by use of both the symmetrical and asymmetrical rate functions are presented in Figs. 2 and 3, respectively. The curves labeled by $\mathrm{P}$ and $\mathrm{Q}$ refer to the periodically and quasi-periodically forced BFs, respectively. The actual, numerically found $\rho-f_{\mathrm{a}}$ dependences being derived by the governing Eq. (1.1) are shown by the solid curves, and the limiting case of the quasi-stationary driven BFs, namely, the average characteristics derivable by the speed Eq. (2.9) are shown by the dashed curves. The presented $\rho-f_{\mathrm{a}}$ dependences evidently demonstrate that the performance of the ratchet-like shuttling of BFs is much lesser pronounced with the quasi-periodic, temporally irregular ac forcing. One can see that the average $\rho-f_{\mathrm{a}}$ characteristics of the quasi-periodically driven BFs are more gently sloped if compared to those being derived by the rigorously periodic forcing $f_{P}(t)$, in the whole interval of the driving amplitudes $f_{\mathrm{a}}$, in both cases the symmetrical and asymmetrical rate functions (compare the curves labeled by $P$ and $Q$ in both figures).

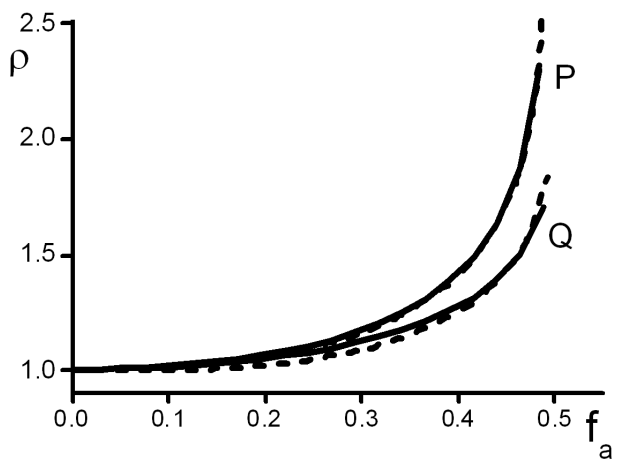

Fig. 2. The acceleration factor $\rho$ of the ac driven front versus the amplitude $f_{\mathrm{a}}$ of the ac force. The curves labeled by $P$ and $Q$ refer to the periodic and quasi-periodic forcing functions, respectively. The actual $\rho-f_{\mathrm{a}}$ dependences that are derived by the governing Eq. (1) are shown by the solid curves; the period of the basic mode of both oscillatory forces was taken as follows, $T=100 \tau_{\mathrm{R}}$. The dashed curves show the average $\rho-f_{\mathrm{a}}$ characteristics being derived by the speed Eq. (2.8). The parameters of the symmetrical rate function used are as follows: $\alpha_{1}=\alpha_{3}=1.0, \alpha_{2}=5.0$, and $h_{\mathrm{R}}=1.05$. The rest of the parameters are: $s_{0} \approx 0.009$ and $\tau_{\mathrm{R}}=1$. The maximal acceleration factors $\rho_{P}\left(f_{\mathrm{M} x}\right)$ and $\rho_{Q}\left(f_{\mathrm{M} x}\right)$ being related to the separate curves $P$ and $Q$ are as follows: 2.5 and 1.7, respectively.

For instance, the maximal acceleration factors being related to the separate curves labeled by $P$ and $Q$ in Fig. 2 are as follows, $\rho_{P}\left(f_{\mathrm{M} x}\right) \approx 2.5$ and $\rho_{Q}\left(f_{\mathrm{M} x}\right) \approx$ 1.7 where the denotation, $\rho_{P, Q}\left(f_{\mathrm{M} x}\right) \equiv \rho_{P, Q}\left(f_{\mathrm{a}}=f_{\mathrm{M} x}\right)$, stands for the acceleration factor taken at the maximal amplitude of the ac forcing, $f_{\mathrm{a}}=f_{\mathrm{M} x}$.

Furthermore, the average $\rho-f_{\mathrm{a}}$ characteristics derivable by the asymmetrical rate functions of the low $R-u$ symmetry demonstrate that both the periodically and quasi-periodically forced BFs can exhibit quite different, dissimilar behavior (see Fig. 3). Indeed, one can see that 


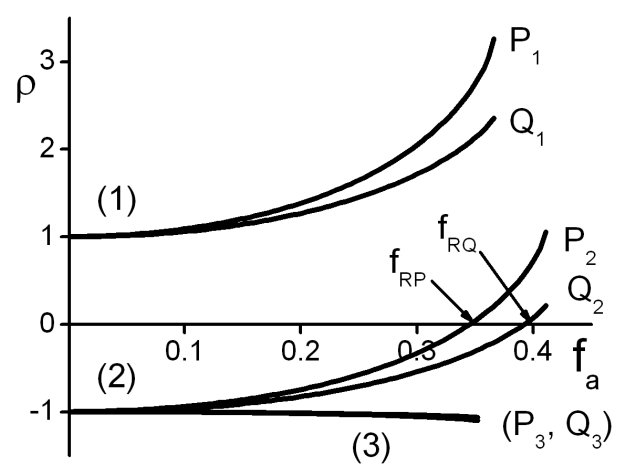

Fig. 3. The same as in Fig. 2 but for the other case of the asymmetrical rate function. The period of the basic mode of the oscillatory forces was taken as follows: $T=100 \tau_{\mathrm{R}}$. The curves labeled by $P_{i}$ and $Q_{i}$ refer to the periodically and quasi-periodically forced BFs, respectively. The "critical" driving amplitudes $f_{\mathrm{a}}=f_{R P}$ and $f_{\mathrm{a}}=f_{R Q}$, at which the reversals of the directed net motion of the periodically and quasi-periodically driven BFs occur, are pointed by the arrows. Parameter values are as follows: $\alpha_{1}=5.0, \alpha_{2}=3.0, \alpha_{3}=1.0, h_{0} \approx$ 1.58 and $\tau_{\mathrm{R}}=1.0 ;($ curves $(1)) h_{\mathrm{R}} \approx 1.74, s_{0} \approx 0.024$ $($ curves $(2)) h_{\mathrm{R}} \approx 1.44, s_{0} \approx-0.024 ;($ curves $(3)) h_{\mathrm{R}} \approx$ $0.55, s_{0} \approx-0.261$.

the presented $\rho-f_{\mathrm{a}}$ dependences of the quasi-periodically driven BFs are more flattened if compared to those derivable with the rigorously periodic forcing $f_{P}(t)$. Thus, the higher strength of the oscillatory force is required to achieve the reversal of the directed net motion of the ac driven $\mathrm{BF}$, with the quasi-periodic, temporally irregular forcing $f_{Q}(t)$ (see curves $P_{2}$ and $Q_{2}$ in Fig. 3). The reversals of the directed net motion of the ac driven BFs are related to the null-points of $\rho-f_{\mathrm{a}}$ characteristics (the null-points, more specifically, the "critical" amplitudes, $f_{\mathrm{a}}=f_{R P}, f_{R Q}$, at which the reversals discussed take place are pointed by arrows in the figure; as before, the labels $P$ and $Q$ in the denotations $f_{R P}$ and $f_{R Q}$ refer to the periodic and quasi-periodic forcing functions, respectively). One can see that both the periodically and quasi-periodically forced BFs will exhibit quite different, dissimilar behavior if the amplitude $f_{\mathrm{a}}$ of the oscillatory forces used is taken within the interval $\left[f_{R P}, f_{R Q}\right]$ : the initial (free) $\mathrm{BF}$, which propagates at the some fixed nonzero velocity $s_{0}$, will propagate, on average, in two opposite directions, depending on the particular ac forcing, either periodic or quasi-periodic one, that was subjected to the front in the system. It should be noted that the average $\rho-f_{\mathrm{a}}$ characteristics that are related to the "backward" running $\left(s_{0}<0\right)$ fronts (see curves $P_{3}$ and $Q_{3}$ in Fig. 3) are very flattened: the spurious drift of $\mathrm{BF}$ practically disappeared in both cases of the periodic and quasi-periodic ac forces, in the whole interval of the driving amplitudes $f_{\mathrm{a}}$. This is in line with the previous findings (see Ref. [10], in particular, Fig. 3 of this reference).

Finally, in closing the discussion of the spurious drift of BFs under slow oscillatory forces we notice that a close proximity of both the actual, numerically found $\rho-f_{\mathrm{a}}$ dependences and those derivable analytically, by use of the speed Eq. (2.9) occurred in both cases of the symmetrical and asymmetrical rate functions. Both $\rho-f_{\text {a }}$ dependences discussed practically coincided if the frequency of the fastest (super-harmonic) mode of the oscillatory forces used was much lesser if compared to the characteristic relaxation rate of the system, namely, when the criterion of the slow driving, $\mu \omega \tau_{\mathrm{R}} \ll 1$, was satisfied. A close proximity discussed is demonstrated by the solid and dashed curves in Fig. 2; the actual, numerically found $\rho-f_{\text {a }}$ dependences are shown by the solid curves, and those being derived by the speed Eq. (2.9) are presented by the dashed curves. One can see that both the numerically found $\rho-f_{\mathrm{a}}$ characteristics and those derivable analytically, within the adiabatic (quasi-stationary) approximation discussed practically coincide, in both cases of the periodically and quasi-periodically forced BFs. Notice that the oscillatory forces that have been used in the numerical derivation of the discussed dependences were taken slow enough, namely, the criterion of the slow driving, $\mu \omega \tau_{\mathrm{R}} \ll 1$, was satisfied (see the figure caption).

Summarizing, we conclude that the temporally irregular oscillations of the unbiased oscillatory forcing shrink the spurious drift of $\mathrm{BF}$ : the performance of ratchet-like shuttling of BFs is much lesser pronounced with the quasi-periodic, temporally irregular ac forcing if compared to that derivable by the rigorously periodic ac force. Let us turn to the ratchet-like shuttling of the quasi-periodically forced BFs being under the action of the fast, rapidly oscillating forces.

\subsection{High-frequency response: self-ordered front under rapidly oscillating force}

In the previous section we have shown that the slow oscillatory forces, the periodic and quasi-periodic one, contribute quite differently to the spurious drift of BFs. As mentioned, the slow oscillatory force usually maximizes the spurious drift of BF. The situation is quite different at the higher driving frequencies $\omega$, beyond the low-frequency interval discussed. The average $\rho-\omega$ characteristics that describe the dependence of the acceleration factor versus the frequency $\omega$ of the basic mode of the oscillatory forces used are presented in Figs. 4 and 5 .

The average characteristics that are derived by use of the symmetrical rate function are shown in Fig. 4 and the other case of the asymmetrical rate function is presented in Fig. 5. The solid curves (labeled by $Q$ ) show the $\rho-\omega$ dependences being derived by the quasi-periodic forcing and the other case of the periodically forced BFs is presented by the dashed curves (labeled by $P$ ) in both figures. One can see that the presented $\rho-\omega$ dependences derivable by both oscillatory forces are similarly shaped: the acceleration factor progressively decreases with the increasing frequency $\omega$, in both cases of the symmetrical and asymmetrical rate functions. Nevertheless, the "efficiency" of both drivers, more specifically, the acceleration factors $\rho$ derivable by both oscillatory forces, the periodic and quasi-periodic one, notably differ. Namely, they 


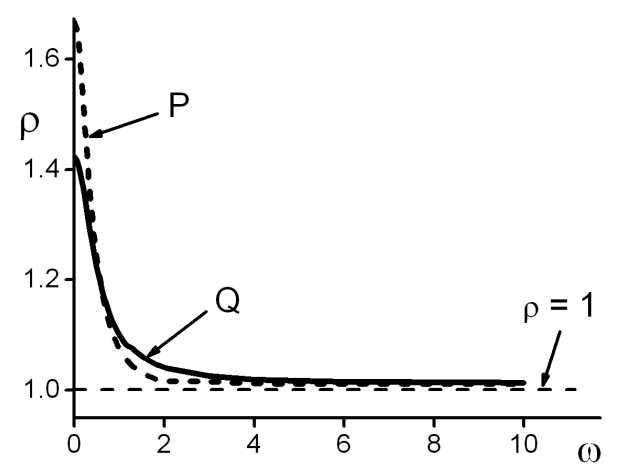

Fig. 4. The acceleration factors of both the periodically and quasi-periodically driven BFs versus the frequency of the fundamental mode of the forcing functions $f_{P}(t)$ and $f_{Q}(t)$ (shown by the dashed $(P)$ and the solid $(Q)$ curves, respectively). The characteristic parameters of the symmetrical rate function are as follows: $\alpha_{1}=\alpha_{3}=1.0, \alpha_{2}=5.0$, and $h_{\mathrm{R}} \approx 1.05$. The rest of the parameters are: $s_{0} \approx 0.009, f_{\mathrm{a}}=0.9 f_{\mathrm{Mx}}$, and $\tau_{\mathrm{R}}=1.0$

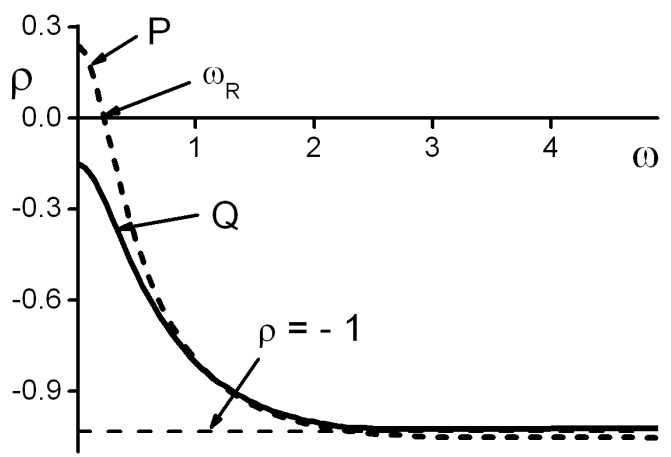

Fig. 5. The same as in Fig. 4 but for the other case of the asymmetrical rate function. The frequency of the ac force, $\omega=\omega_{\mathrm{R}} \approx 0.23$, at which the reversal of the directed net motion of the periodically driven BF takes place is pointed by the arrow. Parameter values of the rate function are as follows: $\alpha_{1}=5.0, \alpha_{2}=$ $3.0, \alpha_{3}=1.0, h_{0} \approx 1.58$, and $h_{\mathrm{R}} \approx 1.44$. The rest of the parameters are: $s_{0} \approx-0.024, f_{\mathrm{a}}=0.9 f_{\mathrm{M} x}$, and $\tau_{\mathrm{R}}=1.0$

deviate more significantly from each other in the low-frequency range, $\omega<\left(\mu \tau_{R}\right)^{-1}$, only: the performance of the ratchet-like shuttling of $\mathrm{BF}$ is much more pronounced with the periodic forcing, in both cases of the symmetrical and asymmetrical rate functions (compare the solid and dashed curves in both Figs. 4 and 5).

By contrast, at the higher frequencies $\omega$, beyond the low-frequency interval discussed, the acceleration factors $\rho$ drivable by both oscillatory forces are close to each other, they practically coincide. Such a dissimilar behavior of both the periodically and quasi-periodically forced BFs within two different frequency intervals discussed may be attributed to the retardation effects in the front dynamics. A close proximity of the discussed $\rho-\omega$ de- pendences in the frequency domain $\omega>\left(\mu \tau_{\mathrm{R}}\right)^{-1}$ demonstrates that the fastest (super-harmonic) mode of both forcing functions $f_{P}(t)$ and $f_{Q}(t)$ is "passive", in terms of the spurious drift discussed, more specifically, the contribution of the super-harmonic mode of both oscillatory forcing functions to the spurious drift of BF is insignificant if the frequency of the super-harmonic mode, $\mu \omega$, exceeds the characteristic relaxation rate of the system, namely, when the relation $\mu \omega \geq \tau_{\mathrm{R}}^{-1}$ is satisfied (for related studies see Refs. $[19,20])$. We remind that both the "spectral content" and the amplitude-to-frequency ratios of the single-harmonic components of both forcing functions used, $f_{P}(t)$ and $f_{Q}(t)$, are very close to each other, they practically coincide.

Let us touch briefly on the reversal behaviour of the directed net motion of the quasi-periodically forced BFs derivable by the asymmetrical rate functions. The mean drift velocity of the ac driven $\mathrm{BF}$ is controllable by adjusting both the amplitude and the frequency of the ac forcing, thus, the occurrence/vanishing of the reversals discussed should be sensitive to both parameters discussed, the frequency and the amplitude of the oscillatory force. Regretfully, the rigorous criteria for the existence (occurrence/vanishing) of the reversals are lacking; the approximate criteria, more specifically, the necessary, but insufficient conditions for the existence of the reversals discussed have been presented in Ref. [10]. Generally speaking, the reversal implies that the average characteristic of the ac driven $\mathrm{BF}$, either $\rho-f_{\mathrm{a}}$ or $\rho-\omega$, has at least one null (zero-point).

As discussed, the average characteristics of the spurious drift of both the periodically and quasi-periodically forced BFs deviate more significantly from each other in the low-frequency range satisfying the relation $\mu \omega<\tau_{\mathrm{R}}^{-1}$, in both cases of the symmetrical and asymmetrical rate functions (see Figs. 4, 5 and 6). The average $\rho-f_{\mathrm{a}}$ and $\rho-\omega$ characteristics derivable by both the periodic and quasi-periodic ac forces become very close to each other, i.e., the peculiarities of the ratchet-like transport derivable by both oscillatory forces discussed will practically coincide if the fastest (super-harmonic) mode of the oscillatory forces becomes "passive", in terms of the spurious drift discussed, namely, when the relation $\mu \omega \gg \tau_{\mathrm{R}}^{-1}$ is satisfied.

The typical $\rho-\omega$ and $\rho-f_{\mathrm{a}}$ dependences being derived by use of the asymmetrical rate functions are presented in Figs. 5 and 6, respectively. They evidently demonstrate that a dissimilar behavior of both the periodically and quasi-periodically forced BFs is observed in the low-frequency domain discussed, only.

In particular, the average $\rho-\omega$ characteristics shown in Fig. 5 demonstrate that the reversal of the unforced $\mathrm{dc}$ motion of BF takes place with the periodic ac force only; the progressively decreasing (decelerated) dc drift of the quasi-periodically driven $\mathrm{BF}$ is observed with the increasing frequency $\omega$.

A dissimilar behavior of both the periodically and quasi-periodically forced BFs is observed in the low- 


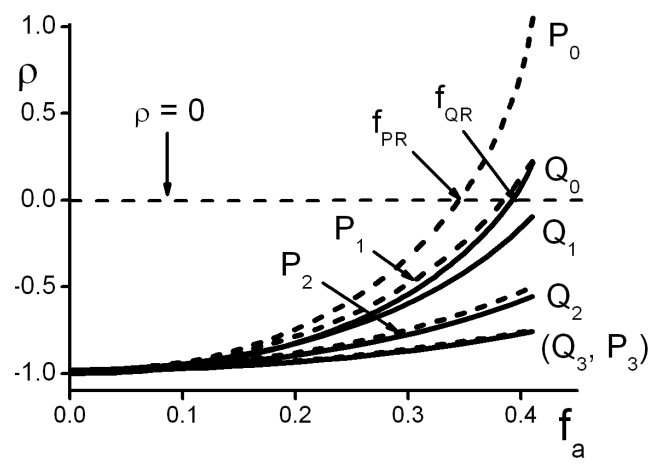

Fig. 6. The acceleration factor of the ac driven front versus the amplitude of the oscillatory force. The average $\rho-f_{\mathrm{a}}$ characteristics of the periodically and quasi-periodically forced BFs are presented by the dashed $\left(P_{j}\right)$ and the solid $\left(Q_{j}\right)$ curves, respectively. The parameters of the asymmetrical rate function are as follows: $\alpha_{1}=5.0, \alpha_{2}=3.0, \alpha_{3}=1.0, h_{0} \approx 1.58$, and $h_{\mathrm{R}} \approx 1.44$. The rest of the parameters are: $s_{0} \approx 0.009$, and $\tau_{\mathrm{R}}=1$. The frequencies $\omega$ of the oscillatory forces being related to the separate pairs of the curves labeled by $\left(P_{0}, Q_{0}\right),\left(P_{1}, Q_{1}\right),\left(P_{2}, Q_{2}\right)$, and $\left(P_{3}, Q_{3}\right)$ are as follows: $\omega \tau_{\mathrm{R}}=0.01,0.30,0.70$, and 1.00 , respectively. Notice that the average $\rho-f_{\mathrm{a}}$ characteristics being derived by both the periodic and quasi-periodic forcing functions that satisfy the relation $\mu \omega \tau_{\mathrm{R}} \geq 1$ are very close to each other (compare the interrelated curves labeled by $\left(P_{2}, Q_{2}\right)$ and $\left(P_{3}, Q_{3}\right)$, respectively).

-frequency domain, $\omega<\left(\mu \tau_{\mathrm{R}}\right)^{-1}$, by tuning the oscillatory frequency $\omega$ : the free $\mathrm{BF}$, which initially propagates at the same fixed velocity $s_{0}$, will propagate, on average, in two opposite directions if the frequency of the oscillatory force, either periodic or quasi-periodic one, being subjected to the front in the system satisfies the relation $\omega<\omega_{\mathrm{R}}$ (the null-point of the average $\rho-\omega$ characteristic, more specifically, the "critical" frequency, $\omega=\omega_{\mathrm{R}}$, at which the reversal of the periodically forced BF takes place is pointed by arrow in Fig. 5). As already noted, such a contrasting, dissimilar behavior of both the periodically and quasi-periodically forced BFs within two different intervals of the frequency $\omega$ may be attributed to the retardation effects in the front dynamics.

The average $\rho-f_{\mathrm{a}}$ characteristics being derived at the different frequencies $\omega$ of the oscillatory forces used confirm this prediction; these are shown in Fig. 6. One can see that the presented $\rho-f_{\mathrm{a}}$ dependences are decreasingly sloped with the frequency $\omega$ of the oscillatory force being increased (compare the curves labeled by $\left(P_{0}, Q_{0}\right)$, $\left(P_{1}, Q_{1}\right),\left(P_{2}, Q_{2}\right)$, and $\left(P_{3}, Q_{3}\right)$ in Fig. 6). This in line with the previous findings (see Refs. $[19,20]$ ): the retardation effects in the front dynamics shrink the spurious drift of BFs. More specifically, the lag time between the ac force $f(t)$ and the speed function $s(t)$ of the ac driven BF increases with the increasing frequency of the ac forcing, as a consequence, the average $\rho-f_{\mathrm{a}}$ characteristics of the spurious drift of BFs become more and more flattened with the oscillatory frequency of the ac force being increased. Indeed, the average $\rho-f_{\mathrm{a}}$ characteristics being derived with the slow oscillatory forces satisfying the relation $\omega<\tau_{\mathrm{R}}^{-1}$ are more steeply sloped (see curves $\left(P_{0}, Q_{0}\right)$ and $\left(P_{1}, Q_{1}\right)$ in Fig. 6): the reversal behavior of the ac driven BFs is observed in the case of the slow oscillatory forces only, when the retardation effects in the front dynamics are insignificant (the reversal-points, i.e., the "critical" amplitudes $f_{P R}$ and $f_{Q R}$, at which the reversals of both the periodically and quasi-periodically driven BFs take place are pointed by arrows in the figure).

In particular, the average $\rho-f_{\text {a }}$ characteristics shown by curves $P_{0}$ and $Q_{0}$ demonstrate that quite different, dissimilar behavior of both periodically and quasi-periodically forced BFs is observed in the low frequency domain discussed, in accord with the previous findings (see Figs. 3 and 5). Namely, they show that both the periodically and quasi-periodically forced BFs will propagate, on average, in the opposite directions if the amplitude $f_{\mathrm{a}}$ of the oscillatory forces, the periodic and quasi-periodic one, was taken within the interval $\left[f_{P R}, f_{Q R}\right]$.

In closing the discussion of the spurious drift of the rapidly driven BFs we stress that the effect of the temporally irregular oscillations of the oscillatory force on the ratchet-like transport of BFs is significant in the low-frequency domain only, when the frequency of the super-harmonic mode, being responsible for the temporal behavior of the oscillatory function, either periodic or quasi-periodic one, is much lesser if compared to the characteristic relaxation rate of the system. At the higher frequencies, when the fastest (super-harmonic) modes of the oscillatory forcing functions, the periodic and quasi-periodic one, become "passive", more specifically, when the fastest mode contributes only insignificantly to the spurious drift of BF, the average characteristics of the ratchet-like transport of both the periodically and quasi-periodically forced BFs practically coincide.

\section{Summary}

The ratchet-like transport of the quasi-periodically forced "bistable" fronts (BFs) joining two states of the different stability in a bistable system of the reaction-diffusion type has been studied within the piecewise linear model of the system, by use of the macroscopic kinetic equation of the reaction kinetics. The quasi-periodic ac force acting on the front in the system was approximated by the biharmonic, temporally irregular forcing function being a superposition of the single-harmonic components (the Fourier modes) that were characterized by the incommensurate, rationally independent frequencies. By considering the response of the self-ordered front to the biharmonic oscillatory forces, both the periodic and quasi-periodic one, we have shown that: (a) The temporally irregular oscillations of the unbiased oscillatory forcing shrink the spurious drift of BFs; the performance of the ratchet-like shuttling of the quasi-periodically forced BFs is much lesser pronounced if compared to that derivable by the rigorously periodic ac force, in both cases of 
the symmetrical and asymmetrical rate functions satisfying the different symmetry. (b) The average characteristics of the ratchet-like transport derivable by the different oscillatory forces, the periodic and quasi-periodic one, deviate more significantly from each other in the low-frequency range of the oscillatory forces, when the frequency of the super-harmonic (fastest) mode of the oscillatory forces used is lesser if compared to the characteristic relaxation rate of the system. By contrast, at the higher frequencies of the ac forces, beyond the low-frequency domain discussed, the average characteristics of the ratchet-like transport of both the periodically and quasi-periodically forced BFs are close to each other. (c) Quite different, dissimilar reversal behavior of the unforced dc motion of both the periodically and quasi-periodically driven BFs is observed (identified) in the low-frequency domain discussed.

\section{Appendix: auxiliary functions of the speed equation}

The auxiliary functions $\varphi\left(s_{\mathrm{A}}\right), \Psi_{S n}\left(s_{\mathrm{A}}\right)$ and $\Phi\left(s_{\mathrm{A}}\right)$ being inherent in the speed Eq. (2.9) are expressed as follows:

$$
\begin{aligned}
& \varphi\left(s_{\mathrm{A}}\right)=\frac{s_{\mathrm{A}} \Phi\left(s_{\mathrm{A}}\right)}{Q_{2}\left(s_{\mathrm{A}}\right)}, \quad \Psi_{S n}\left(s_{\mathrm{A}}\right)=F_{S n} / F_{\mathrm{V}}, \\
& \Phi\left(s_{\mathrm{A}}\right)=\left\{\begin{array}{l}
\arctan \Psi_{T g}\left(s_{\mathrm{A}}\right), \quad \Psi_{T g}\left(s_{\mathrm{A}}\right)>0, \\
\pi-\arctan \left(-\Psi_{T g}\left(s_{\mathrm{A}}\right)\right), \quad \Psi_{T g}\left(s_{\mathrm{A}}\right)<0,
\end{array}\right.
\end{aligned}
$$

with the unknown functions being defined by the relations,

$$
\begin{aligned}
& F_{S n}=Q_{2}\left(s_{\mathrm{A}}\right)\left[\delta_{1} K_{1}\left(s_{\mathrm{A}}\right)-\delta_{3} K_{3}\left(s_{\mathrm{A}}\right)\right], \\
& F_{V}=Q_{2}^{2}\left(s_{\mathrm{A}}\right)+G_{1}^{2}\left(s_{\mathrm{A}}\right), \\
& \Psi_{T g}\left(s_{\mathrm{A}}\right)=F_{S n} / F_{C n}, \\
& F_{C n}=-\left[Q_{2}^{2}\left(s_{\mathrm{A}}\right)+G_{1}\left(s_{\mathrm{A}}\right) G_{3}\left(s_{\mathrm{A}}\right)\right],
\end{aligned}
$$

where

$$
\begin{aligned}
& Q_{2}\left(s_{\mathrm{A}}\right)=\sqrt{1-s_{\mathrm{A}}^{2}}, \\
& K_{1,3}\left(s_{\mathrm{A}}\right)=-s_{\mathrm{A}} \pm \sqrt{r_{1,3}+s_{\mathrm{A}}^{2}}, \\
& G_{1,3}=-s_{\mathrm{A}}+\delta_{1,3} K_{1,3}\left(s_{\mathrm{A}}\right) .
\end{aligned}
$$

The parameters $\delta_{1,3}$ and $r_{1,3}$ in Eqs. (A.3) and (A.5) are given by the following relations: $\delta_{1,3}=\alpha_{2} / \alpha_{1,3}$ and $r_{1,3}=\delta_{1,3}^{-1}$. The boundary conditions $u_{0}(z \rightarrow \mp \infty) \rightarrow u_{1,3}$ that have been discussed in the main text have been used in the derivation of the presented expressions.

\section{References}

[1] F. Julicher, A. Adjari, J. Prost, Rev. Mod. Phys. 69, 1269 (1997); P. Reimann, Phys. Rep. 361, 57 (2002); R. Mallik, P. Gross, Current Biology 14, R971 (2004); P. Hanggi, F. Marchesoni, Rev. Mod. Phys. 81, 387 (2009).
[2] A V. Savin, G.P. Tsironis, Y. Zolotariuk, Phys. Rev. E 56, 2457 (1997); G. Costantini, F. Marchesoni, M. Borromeo, Phys. Rev. E 65, 051103 (2002); M. Salerno, Y. Zolotaryuk, Phys. Rev. E 65, 056603 (2002); M. Salerno, N.R. Quintero, Phys. Rev. E 65, 025602 (2002); S. Flach, Y. Zolotaryuk, A.E. Morishnichenko, M.V. Fistul, Phys. Rev. Lett. 88, 184101 (2002); L. Morales-Molina, N.R. Quintero, F.G. Mertens, A. Sanchez, Phys. Rev. Lett. 91, 234102 (2003); N.R. Quintero, B. Sanchez-Rey, M. Salerno, Phys. Rev. E 72, 016610 (2005); L. Morales-Molina, N.R. Quintero, A. Sanchez, F.G. Mertens, Chaos 16, 013117 (2006).

[3] J. Armero, J.M. Sancho, J. Casademunt, A.M. Lacasta, L. Ramirez-Piscina, F. Sagues, Phys. Rev. Lett. 76, 3045 (1996); J. Armero, J. Casademunt, L. Ramirez-Piscina, J.M. Sancho, Phys. Rev. E 58, 5494 (1998); M.A. Santos, J.M. Sancho, Phys. Rev. E 64, 016129 (2001); Ch.R. Doering, C. Muller, P. Smereka, Physica A 325, 243 (2003).

[4] M. Buttiker, R. Landauer, Phys. Rev. A $\mathbf{2 3}$, 1397 (1981); A.S. Mikhailov, L. Schimansky-Geier, W. Ebeling, Phys. Lett. A 96, 453 (1983); J.M. Sancho, A. Sanchez, Eur. Phys. J. B 16, 127 (2000).

[5] D.J. Kaup, Phys. Rev. B 27, 6787 (1983); L. Schimansky-Geier, Ch. Zulicke, Z. Phys. B, Condens. Matter 82, 157 (1991).

[6] F. Marchesoni, Phys. Rev. Lett. 77, 2364 (1996).

[7] N. Quintero, A. Sanchez, F.G. Mertens, Phys. Rev. E 60, 222 (1999).

[8] M.G. Clerc, C. Falconi, E. Tirapegui, Phys. Rev. Lett. 94, 148302 (2005).

[9] F.G. Bass, R. Bakanas, Europhys. Lett. 53, 444 (2001).

[10] R. Bakanas, Phys. Rev. E 69, 016103 (2004).

[11] R. Bakanas, Phys. Rev. E 71, 026201 (2005).

[12] R. Bakanas, Phys. Rev. E 78, 046202 (2008).

[13] M. Bleck, E. Goldobin, M. Neuhaus, M. Siegel, R. Kleiner, D. Koelle, Phys. Rev. Lett. 95, 090603 (2005).

[14] S. Flach, Y. Zolotaryuk, A.E. Miroshnichneko, M.V. Fistul, Phys. Rev. Lett. 88, 184101 (2002); M. Salerno, Y. Zolotaryuk, Phys. Rev. E 65, 056603 (2002).

[15] S. Denisov, S. Flach, A.A. Ovchinnikov, O. Yevtushenko, Y. Zolotaryuk, Phys. Rev. E 66, 041104 (2002); R. Gommers, S. Denisov, F. Renzoni, Phys. Rev. Lett. 96, 240604 (2006).

[16] J.P. Gleeson, Physica A 388, 277 (2009); P.R. Kramer, O. Kurbanmuradov, K. Sabelfeld, J. Comp. Phys. 226, 897 (2007).

[17] M. Locher, D. Cigna, E.R. Hunt, Phys. Rev. Lett. 80 , 51212 (1998).

[18] R. Bakanas, Nonlinearity 16, 313 (2003).

[19] A. Raguotis, F. Ivanauskas, R. Bakanas, Phys. Scr. 74, 629 (2006).

[20] R. Bakanas, A. Raguotis, F. Ivanauskas, Phys. Scr. 77, 055003 (2008). 